\title{
Atividade antifúngica do óleo essencial de Origanum vulgare frente a Malassezia pachydermatis
}

\author{
[Antifungal activity of Origanum vulgare essential oil against Malassezia pachydermatis] \\ R. Santin ${ }^{1}$, C. Giordani ${ }^{2}$, I.M. Madrid ${ }^{3}$, C.B. Matos $^{2}$, R.A. Freitag ${ }^{2}$, M.C.A. Meireles ${ }^{2}$, \\ M.B. Cleff ${ }^{2}$, J.R.B. Mello \\ ${ }^{1}$ Programa de pós-graduação - Universidade Federal do Rio Grande do Sul - Porto Alegre, RS \\ ${ }^{2}$ Programa de pós-graduação - Universidade Federal de Pelotas - Pelotas, RS \\ ${ }^{3}$ Prefeitura Municipal de Pelotas - Pelotas, RS
}

\begin{abstract}
RESUMO
Objetivou-se com este estudo avaliar a atividade antifúngica in vitro do óleo essencial de Origanum vulgare frente a isolados clínicos de Malassezia pachydermatis. As folhas secas de O. vulgare foram adquiridas de distribuidor comercial com certificado de qualidade e origem e encaminhadas para extração do óleo essencial e cromatografia. Para realização do teste in vitro, foi utilizada a técnica de microdiluição em caldo (CLSI M27A3) com modificações para fitofármacos e M. pachydermatis. O óleo essencial de orégano foi testado nas concentrações de 28 a $0,87 \mathrm{mg} / \mathrm{mL}$ diluído em caldo Sabouraud com $1 \%$ de tween 80. Todos os isolados foram testados em duplicata. Na análise cromatográfica do óleo essencial, foram identificados 12 compostos, sendo timol, $\alpha$-terpineno e 4-terpineol os compostos majoritários. A CIM e a CFM dos 42 isolados de $M$. pachydermatis variaram de $\leq 0,87$ a $7 \mathrm{mg} / \mathrm{mL}$, com valores de $\mathrm{CIM}_{50}$ e $\mathrm{CIM}_{90}$ de 1,18 e $3,28 \mathrm{mg} / \mathrm{mL}$, respectivamente. Com este estudo foi possível concluir que $M$. pachydermatis é sensível ao óleo essencial de orégano mesmo em concentrações baixas. Dessa maneira, o óleo essencial de orégano apresenta-se como promissor na bioprospecção de novos fármacos para o tratamento das otites e dermatites na clínica de pequenos animais.
\end{abstract}

Palavras-chave: cão, extratos vegetais, leveduras, orégano, suscetibilidade

\begin{abstract}
The aim of this study was to evaluate the in vitro antifungal activity of essential oil of Origanum vulgare against clinical isolates of Malassezia pachydermatis. The dried leaves of $\mathrm{O}$. vulgare were purchased from a commercial distributor with certified quality and origin and referred for essential oil extraction and chromatography. The technique for in vitro testing was microdilution (CLSI M27A3) with modifications to phytochemicals and M. pachydermatis. The essential oil of O. vulgare was tested at concentrations from 28 to $0.87 \mathrm{mg} / \mathrm{mL}$ in Sabouraud broth diluted with $1 \%$ of tween 80 . All isolates were tested in duplicate. In the chromatographic analysis of the essential oil 12 compounds were identified, and thymol, $\alpha$-terpinene, 4-terpineol were the major compounds. The MIC and the MFC of the 42 isolates of $\mathrm{M}$. pachydermatis ranged from $\leq 0.87$ to $7 \mathrm{mg} / \mathrm{mL}$ with $\mathrm{MIC}_{50}$ and $\mathrm{MIC}_{90}$ values of 1.18 and 3.28 $\mathrm{mg} / \mathrm{mL}$, respectively. With this study it was concluded that $\mathrm{M}$. pachydermatis is sensible to $\mathrm{O}$. vulgare essential oil even at low concentrations. Thus, the essential oil of $\mathrm{O}$. vulgare is presented as bioprospecting in the promising new drugs for the treatment of otitis and dermatitis in small animal clinic.
\end{abstract}

Keywords: dog, plant extracts, yeasts, oregano, susceptibility

Recebido em 6 de fevereiro de 2013

Aceito em 1 de outubro de 2013

E-mail: seminhavet@yahoo.com.br 


\section{INTRODUÇÃO}

A utilização de plantas na terapêutica acompanha a evolução da humanidade, de forma que o uso popular desses recursos no tratamento de doenças é descrito há muitos anos, mesmo sem confirmação científica. É importante salientar que cerca de $80 \%$ da população de baixa renda não tem acesso à assistência farmacêutica. Assim, as plantas medicinais representam uma fonte viável para o tratamento de enfermidades (Veiga Junior et al. 2005).

Atualmente, políticas federais de incentivo às pesquisas, visando ao uso seguro e eficaz de fitoterápicos, têm sido implementadas. Nesse sentido, a ANVISA (Agência Nacional de Vigilância Sanitária) organizou um manual para divulgação de diversas espécies com potencial terapêutico bem descrito e aceito cientificamente, principalmente para serem utilizadas pelo SUS (Sistema Único de Saúde) (Brasil, 2011).

No contexto atual, destaca-se o Origanum vulgare L., popularmente conhecido como orégano, uma planta aromática utilizada principalmente na culinária que tem seu valor medicinal reconhecido. Além das folhas, usadas no tratamento popular, o óleo essencial tem demonstrando eficácia em pesquisas, principalmente como antimicrobiano (Lambert et al., 2001; Manohar et. al. 2001; Souza et al. 2005; Cleff et al., 2010a; Cleff et al., 2010b).

A malasseziose está entre as micoses mais diagnosticadas em pequenos animais, sendo a Malassezia pachydermatis de grande importância nas otites e dermatites (Nobre et al.,1998; Nascente et al., 2004). O gênero Malassezia possui 14 espécies, todas lipodependentes (Cabañes et al., 2011), com exceção da $M$. pachydermatis, única não-lipodependente e a mais envolvida na casuística dermatológica da clínica de pequenos animais (Campbell et al., 2010). Alterações no microambiente do conduto auditivo, como aumento da temperatura, umidade e substrato, favorecem o aumento do número de células e a passagem da levedura da forma comensal para a parasitária ou patogênica. O mesmo acontece nas dermatites, em que o desequilíbrio na imunidade do hospedeiro é um dos principais fatores desencadeantes da malasseziose (Nobre et al.,1998; Nascente et al., 2004). Além disso, tem sido demonstrada a importância dessa levedura em saúde pública, visto que a mesma já foi isolada e associada à formação de biofilmes, que podem atuar como fonte de infecções hospitalares em pacientes imunocomprometidos, principalmente neonatos (Canizzo et al., 2007). A resistência de $M$. pachydermatis aos antifúngicos comumente utilizados no tratamento da enfermidade já foi demonstrada por Fera et al. (2009), Jesus et al. (2011) e Nijima et al. (2011).

Tendo em vista a grande importância do uso de plantas medicinais na Veterinária e o papel de $M$. pachydermatis como um dos principais agentes de otites externas e dermatites secundárias em cães, objetivou-se, com este trabalho, avaliar a atividade antifúngica in vitro do óleo essencial de $O$. vulgare frente a isolados clínicos de $M$. pachydermatis.

\section{MATERIAL E MÉTODOS}

Para o estudo da atividade antifúngica, o $O$. vulgare (orégano) foi adquirido de distribuidor comercial com certificado de qualidade e origem. Para obtenção do óleo essencial, as folhas secas foram submetidas à extração com arraste de vapor em aparelho Clevenger, segundo a Farmacopeia Brasileira IV, durante 4 horas. Depois, o óleo obtido foi seco com sulfato de sódio anidro p.a, armazenado em frasco âmbar e mantido sob refrigeração até a utilização.

Realizou-se a análise cromatográfica em equipamento CG/FID (Schimadzu, modelo 2010) equipado com uma coluna de sílica DB-5 (30m x $0,25 \mathrm{~mm} \times 0,25 \mu \mathrm{m})$, com temperatura inicial de $40^{\circ} \mathrm{C}$, ocorrendo um aumento na taxa de $2^{\circ} \mathrm{C} \mathrm{min}{ }^{-}$ ${ }^{1}$ até atingir $145^{\circ} \mathrm{C}$. A partir dessa temperatura, a taxa foi de $10^{\circ} \mathrm{C} \min ^{-1}$ até atingir $280^{\circ} \mathrm{C}$, permanecendo nessa temperatura por $10 \mathrm{~min}$; $\mathrm{Td}$ $=280^{\circ} \mathrm{C}$; Tinj $=280^{\circ} \mathrm{C} ;$ Tcol $=40^{\circ} \mathrm{C}$; Split $=$ 1:50.

Foram preparadas soluções do óleo a $5.000 \mathrm{mg}^{-1}$ em hexano e dos padrões cromatográficos a $40 \mathrm{mg} \mathrm{L}^{-1}$ ( $\alpha$-pineno, canfeno, $\beta$-pineno, mirceno, $\alpha$-terpineno, $\quad$-cimeno, limoneno, 1,8-cineol, terpinoleno, linalol, 4terpineol, $\alpha$-terpineol, timol e carvacrol), das quais foram injetadas no cromatógrafo em volume de $1 \mu \mathrm{L}$. Os constituintes foram identificados por comparação entre o tempo de retenção dos padrões e da amostra. 
Para realização do teste do óleo essencial de $O$. vulgare, foi utilizada a técnica de microdiluição em caldo de acordo com o documento M27A3 do CLSI (Clinical and Laboratory Standars Institute) com modificações para fitofármacos e M. pachydermatis (Cleff et al., 2010a).

Foram estudados 42 isolados de $M$. pachydermatis provenientes de casos clínicos de otites $(n=35)$ e de dermatites $(n=7)$ em cães que se encontravam estocados na micoteca do Centro de Diagnóstico e Pesquisa em Micologia Veterinária da UFPel. Os inóculos foram preparados a partir de colônias jovens (48h) em ágar Sabouraud dextrose acrescido de cloranfenicol. As colônias foram suspensas em solução salina estéril, homogeneizadas e ajustadas em espectrofotômetro com comprimento de onda $530 \mathrm{~nm}$ e transmitância entre 60-65\%. A partir dessa solução, foi realizada uma diluição de 1:50 em solução salina estéril e, em seguida, uma diluição de 1:20 em meio Sabouraud líquido, a qual foi dispensada em alíquotas de $100 \mu \mathrm{L}$ nos poços das microplacas. O óleo essencial de orégano foi testado nas concentrações de 28 a $0,87 \mathrm{mg} / \mathrm{mL}$ diluído em caldo Sabouraud com $1 \%$ de Tween 80. Todos os isolados foram testados em duplicata.
Depois de preenchidas, as microplacas foram incubadas a $35^{\circ} \mathrm{C}$ por $72 \mathrm{~h}$ para realização da leitura da Concentração Inibitória Mínima (CIM). Posteriormente, realizou-se a transferência de $10 \mu \mathrm{L}$ de cada poço para placas de Petri contendo ágar Sabouraud dextrose acrescido de cloranfenicol, as quais foram incubadas a $35^{\circ} \mathrm{C}$ por $72 \mathrm{~h}$ para leitura da Concentração Fungicida Mínima (CFM).

Os dados da CIM e CFM foram expressos pela média das duplicatas e relacionados como valores em que $50 \%\left(\mathrm{CIM}_{50}\right)$ e $90 \%\left(\mathrm{CIM}_{90}\right)$ dos isolados foram inibidos. M. pachydermatis foi classificada como sensível (S) quando CIM da amostra $<\mathrm{CIM}_{50}$, sensibilidade intermediária (I) quando $\quad \mathrm{CIM}_{50}<\mathrm{CIM} \quad$ da $\quad$ amostra $\leq \mathrm{CIM}_{90} \quad \mathrm{e}$ resistente (R) quando CIM da amostra $>\mathrm{CIM}_{90}$, conforme realizado por Nascente et al. (2003), que utilizaram antifúngicos convencionais.

\section{RESULTADOS}

Os resultados obtidos através do teste de microdiluição em caldo confirmam o efeito antifúngico do óleo essencial de orégano frente a isolados clínicos de $M$. pachydermatis. A análise cromatográfica do óleo essencial identificou 12 constituintes (Fig. 1), sendo timol (pico 13), $\alpha$ terpineno (pico 5) e 4-terpineol (pico 11) os compostos majoritários.

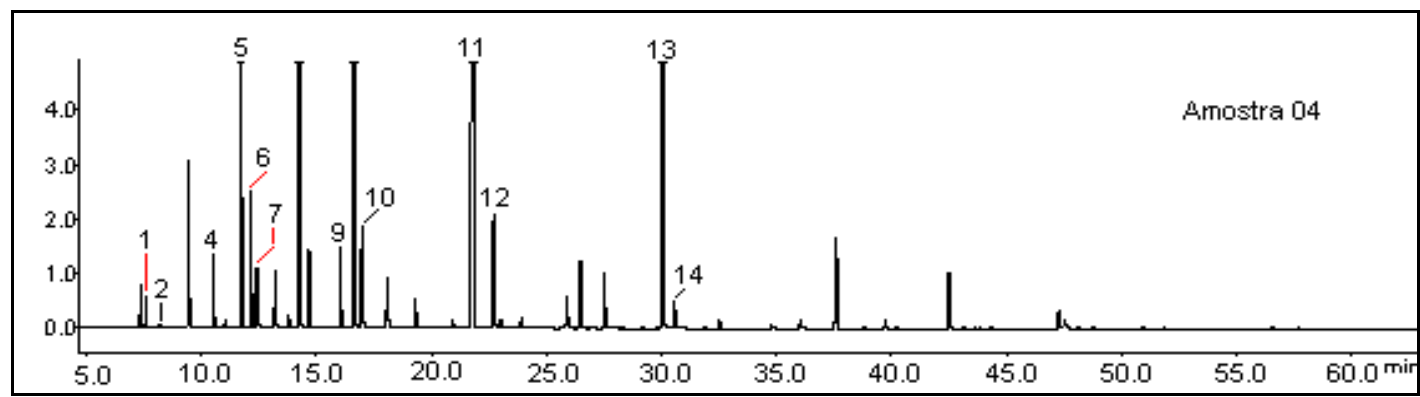

Figura 1 - Padrões utilizados e os picos dos constituintes encontrados na cromatografia gasosa da amostra de Origanum vulgare, sendo: 1- $\alpha$-pineno; 2- canfeno; 3 - $\beta$-pineno; 4- mirceno; 5 - $\alpha$-terpineno; 6 - p-cimeno; 7 Limoneno; 8- 1,8-cineol; 9- terpinoleno; 10- linalol; 11- 4-terpineol; 12- $\alpha$-terpineol; 13- timol; 14- carvacrol.

A CIM e a CFM dos 42 isolados de $M$. pachydermatis variaram de $\leq 0,87$ a $7 \mathrm{mg} / \mathrm{mL}$, com valores de $\mathrm{CIM}_{50}$ e $\mathrm{CIM}_{90}$ de 1,18 e $3,28 \mathrm{mg} / \mathrm{mL}$, respectivamente. A CIM e CFM do óleo de orégano para os isolados de dermatite foram de $1,75 \mathrm{mg} / \mathrm{mL}$ para três isolados, $\leq 0,87 \mathrm{mg} / \mathrm{mL}$ para dois isolados, $3,5 \mathrm{mg} / \mathrm{mL}$ para um isolado e apenas um isolado com diferença de CIM e CFM, respectivamente $\leq 0,87$ e $1,75 \mathrm{mg} / \mathrm{mL}$. Os resultados com os valores de CIM e CFM estão distribuídos na Figura 2.

Quanto à sensibilidade, os isolados foram classificados em sensíveis, intermediários e resistentes, de acordo com a CIM $_{50}$ e CIM $_{90}$ (Tab. 1). 


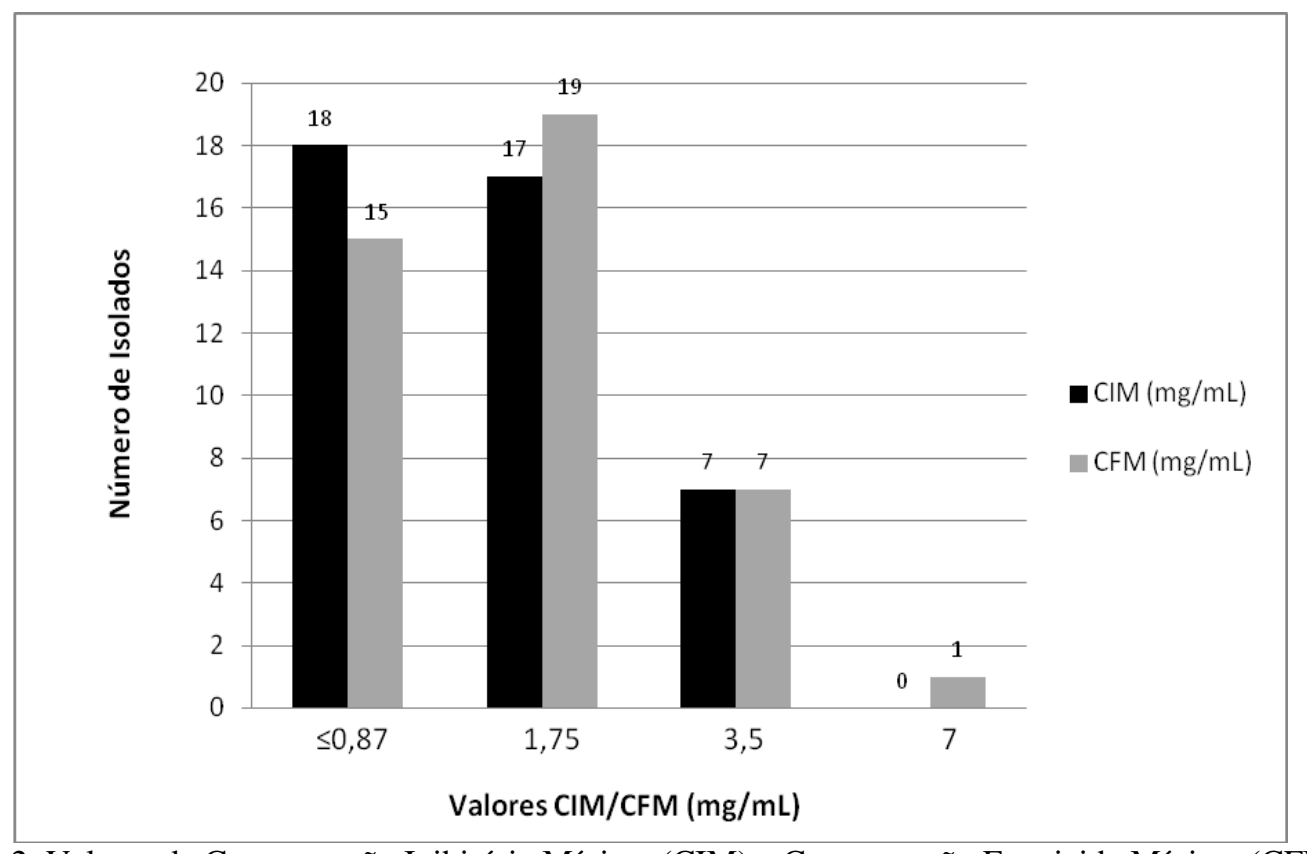

Figura 2. Valores da Concentração Inibitória Mínima (CIM) e Concentração Fungicida Mínima (CFM) do óleo essencial de orégano frente a diferentes isolados clínicos de M. pachydermatis provenientes de otite e dermatite em cães.

Tabela 1. CIM do óleo essencial de orégano avaliando a sensibilidade de $M$. pachydermatis isoladas de cães com otite externa e dermatite através do método de microdiluição em caldo

\begin{tabular}{cccc}
\hline & \multicolumn{3}{c}{ Microdiluição em caldo $(\mathrm{mg} / \mathrm{mL})$} \\
\cline { 2 - 4 } Origanum vulgare & $\mathrm{S}$ & $\mathrm{C}$ & $\mathrm{R}$ \\
& $\mathrm{n}(\%)$ & $\mathrm{n}(\%)$ & $\mathrm{n}(\%)$ \\
\cline { 2 - 4 } & $<1,18$ & 1,75 & $>3,28$ \\
& $18(42,86)$ & $17(40,48)$ & $7(16,66)$ \\
\hline
\end{tabular}

S: sensível; I: Intermediária; R: Resistente

\section{DISCUSSÃO}

A busca por alternativas para a terapêutica da malasseziose justifica-se, uma vez que tem sido descrita a ocorrência de resistência antifúngica por parte dessa levedura, além do aumento de casos crônicos e recidivantes (Machado et al., 2003; Santos et al., 2008; Fera et al., 2009), sendo reconhecida a importância clínica de $M$. pachydermatis, principalmente nos casos de otite externa em pequenos animais (Nobre et al., 1998; Leite et al., 2003; Nascente et al., 2004). Além disso, $M$. pachydermatis tem se mostrado sensível a diferentes extratos vegetais e óleos essenciais de diversos produtos naturais, inclusive em concentrações baixas (Cardoso et al., 2010; Lee e Lee, 2010; Lozina et al., 2010), demonstrando a atividade promissora dos produtos naturais frente ao agente.
Os dados obtidos na análise cromatográfica do óleo estão de acordo com a literatura, sendo que os fenóis, como carvacrol, timol, $\gamma$-terpeno e $p$ cimeno, podem alcançar entre $80,2 \%$ a $98 \%$ da composição total do óleo de O. vulgare (Simões et al., 2003; Cleff, 2008). O timol foi um dos principais componentes isolados do óleo essencial estudado, concordando com os achados de Pistelli et al. (2012), cujo principal constituinte de $O$. vulgare foi o timol e com menores quantidades de p-cimeno. $\mathrm{O}$ timol e o carvacrol têm sido considerados como marcadores, pois são os componentes que identificam o $O$. vulgare, conforme foi encontrado por Cleff (2008), que avaliou a composição química de oito amostras do óleo essencial de orégano e identificou os compostos, como timol, carvacrol, $\alpha$-pineno e 4-terpineol, que variaram em suas concentrações. 
Os constituintes 4-terpineol e $\alpha$-terpineno têm sido descritos como responsáveis pela ação antimicrobiana do orégano (Lambert et al., 2001; Ultee et al., 2002; Chami et al., 2004). Dessa forma, provavelmente essas altas concentrações contribuíram para os resultados de CIM e CFM para M. pachydermatis. Além disso, é possível que núcleos aromáticos, contendo um grupo polar, possam fazer ligações de hidrogênio com os sítios ativos de enzimas microbianas, favorecendo essa atividade (Lambert et al., 2001; Ultee et al., 2002).

Dentre os fármacos mais utilizados para as otites e dermatites, destacam-se os azóis, sendo o cetoconazol largamente utilizado na clínica de pequenos animais (Nobre et al., 2002). Entretanto, fatores como o uso inadequado dos produtos comerciais que, muitas vezes, são utilizados indiscriminadamente, além da dose, frequência e tempo inadequado, podem estar relacionados com o surgimento de isolados resistentes (Machado et al., 2003; Fera et al., 2009; Nijima et al., 2011).

A ampla variação dos resultados (CIM e CFM $\leq 0,87$ a $7 \mathrm{mg} / \mathrm{mL}$ ) para o óleo de orégano frente aos isolados considerados resistentes em nosso estudo pode estar relacionada à diversidade dos casos clínicos, incluindo quadros agudos e crônicos. Neste caso, provavelmente resultando em relação inversa entre resistência e evolução do quadro clínico, já que os isolados provenientes de casos crônicos e recidivantes podem apresentar valores de CIM superiores.

A atividade antifúngica de $O$. vulgare tem sido descrita frente a diversos fungos, especialmente leveduras do gênero Candida (Manohar et al., 2001; Chami et al., 2004; Cleff et al., 2010a; Cleff et al., 2010b). Porém, estudos com espécies de Malassezia ainda são insipientes, principalmente utilizando isolados provenientes de animais. Em contrapartida, alguns autores vêm utilizando extratos vegetais em isolados de $M$. pachydermatis, incluindo extratos de $O$. vulgare (Prestes et al., 2008; Cleff et al., 2010a;
Galuppi et al., 2010; Lee e Lee, 2010; Pistelli et al., 2012). Esses estudos divergem em vários aspectos, como origem do óleo essencial e composição química, origem dos isolados e teste de sensibilidade utilizado.

O óleo essencial de orégano utilizado no presente estudo apresentou CIM e CFM $\leq 0,87$ a $7 \mathrm{mg} / \mathrm{mL}$, valores considerados baixos para os isolados de M. pachydermatis, corroborando outros autores (Prestes et al., 2008; Rusenova e Parvanov, 2009; Galuppi et al., 2010) em relação à sensibilidade da levedura ao óleo essencial. E ainda, Cleff et al. (2010a) obtiveram valores entre 0,015 a $0,001 \%$ menores que os encontrados neste estudo. Já valores muito semelhantes foram observados por Prestes et al. (2008) e Rusenova e Parvanov (2009), que verificaram atividade antifúngica entre $0,06 \% \mathrm{e}$ $0,25 \%$ do óleo essencial de orégano. Segundo Galuppi et al. (2010), o óleo essencial de orégano está entre os mais eficientes quando comparados aos 23 óleos testados em seu estudo frente a diferentes espécies de Malassezia. Para Pistelli et al. (2012), o óleo essencial de orégano apresentou CIM de $0,8 \%$, valor este superior ao encontrado em nosso estudo.

\section{CONCLUSÃO}

Com este estudo foi possível concluir que $M$. pachydermatis é sensível ao óleo essencial de orégano mesmo em concentrações baixas. Dessa maneira, o óleo essencial de orégano apresentase como promissor na bioprospecção de novos fármacos para $\mathrm{o}$ tratamento das otites $\mathrm{e}$ dermatites na clínica de pequenos animais.

\section{AGRADECIMENTOS}

Os autores agradecem à Fundação de Amparo à Pesquisa do Rio Grande do Sul (Processo 11/1225-0) pelo financiamento do projeto. À CAPES e ao CNPq pelas bolsas de estudo. 


\section{REFERÊNCIAS}

BRASIL. Agência Nacional de Vigilância Sanitária. Formulário de Fitoterápicos da Farmacopeia Brasileira/Agência Nacional de Vigilância Sanitária. Brasília: ANVISA, 2011. $126 \mathrm{p}$.

CABAÑES，F.J.; VEJA，S.; CASTELL， G. Malassezia cuniculi sp. nov., a novel yeast species isolated from rabbit skin. Med. Mycol., v.49, p.40-48, 2011.

CAMPBELL, J.J.; COYNER, K.S.; RANKIN, S.C. et al. Evaluation of fungal flora in normal and diseased canine ears. Vet. Dermatol., v.21, p.619-25, 2010.

CANIZZO, F.T.; ERASO, E.; EZKURRA, P.A. et al. Biofilm development by clinical isolates of Malassezia pachydermatis. Med. Mycol., v.45, p.357-361, 2007.

CARDOSO, R.L.; MABONI, F.; MACHADO, G. et al. Antimicrobial activity of propolis extract against Staphylococcus coagulase positive and Malassezia pachydermatis of canine otitis. Vet. Microbiol., v.142, p.432-434, 2010.

CHAMI, N.; CHAMI, F.; BENNIS, S. et al. Antifungal Treatment With Carvacrol and Eugenol of Oral Candidiasis in Immunosuppressed Rats. Braz. J. Infect. Dis., v.8, p.217-226, 2004.

CLEFF, M.B. Avaliação da atividade antifúngica do óleo essencial de Origanum vulgare L. frente a fungos de importância em veterinária com ênfase em Candida spp. 2008. 114f. Tese (Doutorado em Ciências Veterinárias) - Faculdade de Veterinária, Universidade Federal do Rio Grande do Sul, Porto Alegre/RS.

CLEFF, M.B.; MEINERZ, A.R.M; FARIA, R.O. et al. Atividade inibitória do óleo essencial de orégano em fungos de importância médica e veterinária. Arq. Bras. Med. Vet. Zootec., v.62, p.1291-1294, 2010a.

CLEFF, M.B.; MEINERZ, A.R.M.; XAVIER, M. et al. In vitro susceptibility of Origanum vulgare essential oil against Candida species. Braz. J. Microbiol. (Impresso), v.41, p.116-123, 2010b.

CLSI M27A3. Reference Method for Broth Dilution Antifungal Susceptibility Testing of Yeasts. Approved Standard-Third Edition, 2008.
FERA, M.T.; CAMERA, E.L.C.; DE SARRO, A. New triazoles and echinocandins: mode of action, in vitro activity and mechanisms of resistance. Expert. Rev. Anti. Infect. Ther., v.7, p.981-998, 2009.

GALUPPI, R.; AURELI, S.; BONOLI, C. et al. Effectiveness of essential oils against Malassezia spp.: comparison of two in vitro tests. Mikol. Lek., v.17, p.79-84, 2010.

JESUS, F.P.K.; LAUTERT, C.; ZANETTE, R.A. et al. In vitro susceptibility of fluconazolesusceptible and -resistant isolates of Malassezia pachydermatis against azoles. Vet. Microbiol., v.152, p.161-164, 2011.

LAMBERT, R.J.W.; SKANDAMIS， P.N.; COOTE, P.J. A Study of the minimum inhibitory concentration and mode of action of oregano essencial oil, thymol and carvacrol. J. Appl. Microbiol., v.91, p.453-462, 2001.

LEE, J.; LEE, J. Inhibitory effect of Plant Essential Oils on Malassezia pachydermatis. J. Appl. Biol. Chem., v.53, p.184-188, 2010.

LEITE, C.A.L.; ABREU, V.L.V.; COSTA, G.M. Frequência de Malassezia pachydermatis em otite externa de cães. Arq. Bras. Med. Vet. Zootec., v.55, p.102-104, 2003.

LOZINA, L.A.; PEICHOTO, M.E.; BOEHRINGER, S.I. et al. Efficacy of Argentine propolis formulation for topical treatment of canine otitis externa. Arq. Bras. Med. Vet. Zootec., v.62, p.1359-1366, 2010

MACHADO, M.L.S.; APPELT, C.E.; FERREIRO, L. et al. Otites e dermatites por Malassezia spp. em cães e gatos. Clin. Vet., v.44, p.27-34, 2003.

MANOHAR, V.; INGRAM, C.; GRAY, J. et al. Antifungal activities of origanum oil against Candida albicans. Mol Cel. Biochem., v.228, p.111-117, 2001.

NASCENTE, P.S.; NOBRE, M.O.; MEINERZ, A.R.M. et al. Ocorrência de Malassezia pachydermatis em cães e gatos. Rev. Bras. Med. Vet., v.26, p.79-82, 2004.

NASCENTE, P.S.; NOBRE, M.O; SCHUCH, L.F. et al. Evaluation of Malassezia pachydermatis antifungal susceptibily using two different methods. Braz. J. Microbiol. (Impresso), v.34, p.359-362, 2003. 
NIJIMA, M.; KANO, R.; NAGATA, M. et al. An azoleresistant isolate of Malassezia pachydermatis. Vet. Microbiol., v.149, p.288290, 2011.

NOBRE, M.O.; MEIRELES, M.C.A.; GASPAR, L.F. et al. Malassezia pachydermatis e outros agentes infecciosos nas otites externas e dermatites em cães. Cienc. Rural, v.28, p.447$452,1998$.

NOBRE, M.O.; NASCENTE, P.S.; MEIRELES, M.C.A. et al. Drogas antifúngicas para pequenos e grandes animais. Cienc. Rural, v.32, p.175184, 2002.

PISTELLI, L.; MANCIANTI, F.; BERTOLI, A. et al. Antimycotic activity of some aromatic plants essential oils against canine isolates of Malassezia pachydermatis: an in vitro assay. Open Mycol. J., v.6, p.17-21, 2012.

PRESTES, L.S.; SCHUCH, L.F.D.; MEIRELES, M.C.A. et al. Actividad de extractos de orégano y tomillo frente a microorganismos asociados con otitis externa. Rev. Cub. Plant. Med., v.13, p.4-8, 2008.

RUSENOVA, N.; PARVANOV, P. Antimicrobial activities of twelve essential oils against microorganisms of veterinary importance. Trak. J. Sci., v.7, p.37-43, 2009.
SANTOS , J.A.; MARTINS, L.A. Atividade in vitro de antifúngicos frente a isolados de Malassezia spp. de animais atendidos no hospital veterinário da Unipar. Arq. Cienc. Vet. Zool., v.11, p.175-178, 2008.

SIMÕES, C.M.O.; SCHENKEL, E.P.; GOSMANN, G. et al. (Ed) Farmacognosia da planta ao medicamento. Porto Alegre/ Florianópolis: Editora da UFRGS/Editora da UFSC, 2003. 1102p.

SOUZA, E.L.; STAMFORD, T.L.M.; LIMA, E.O. et al. Orégano (Origanum vulgare L., Lamiaceae): uma especiaria como potencial fonte de compostos antimicrobianos. Rev. Hig. Alim., v.19, p.40-45, 2005.

ULTEE, A.; SMID, E.J. Influence of carvacrol on growth and toxin production by Bacillus cereus. J. Food Microbiol., v.64, p.373-378, 2001.

VEIGA Jr., V.F.; PINTO, A.; MACIEL, M.A.M. Plantas Medicinais: Cura segura?. Química Nova (Impresso), v.28, p.519-528, 2005. 